\title{
Properties of axial surface waves along dielectrically coated conducting cylinders
}

\author{
U. Siart, S. Adrian, and T. Eibert \\ Lehrstuhl für Hochfrequenztechnik, Technische Universität München, 80290 München, Germany \\ Correspondence to: U. Siart (uwe.siart@tum.de)
}

\begin{abstract}
In this paper the fundamental properties of surface waves along conducting cylinders with and without dielectric coating are investigated for cylinder diameters in the centimeter range and frequencies in the gigahertz range and higher. Analytical results for the phase constant and attenuation versus the cylinder radius are derived and cutoff frequencies of various TE, TM, and hybrid waves are computed. The radial power distribution is computed in order to investigate the spatial extent of the wave fields.
\end{abstract}

\section{Introduction}

Electromagnetic wave propagation along cylinders has been studied in the past. Pioneering work was done by Sommerfeld, Hondros, and Goubau from the beginning to mid of last century. In recently emerging application fields for highfrequency measurement and monitoring systems the properties of surface waves along cylinders with dielectric coating are of particular significance. Exceptionally because of the availability of frequencies for industrial, scientific, and medical applications (ISM) in the gigahertz range a study of cylindrical surface waves in the high-frequency limit where the diameter of the guiding structure is no longer small compared to wavelength is eligible. An exemplary application is the monitoring of surface condition and detracting deposits in ductwork and piping under harsh conditions where ultrasound or optical techniques are inapplicable. A possible configuration for monitoring the surface condition of metal pipes by launching surface waves is suggested in Fig. 1. Conical horns may be used as wave launchers as already presented by Goubau (1951). In case there is a metal wall dielectric windows are suitable to provide through-wall transition. A second horn at the end of the pipe receives the wave. From a transmission and reflection measurement the propagation parameters of the surface wave along the pipe can be recon-

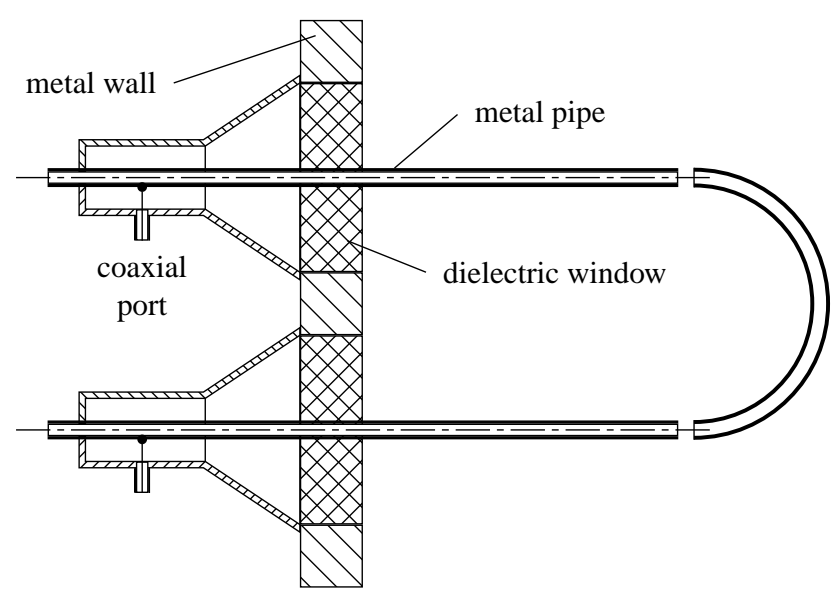

Fig. 1. Example configuration: monitoring the surface condition of metal pipes by launching surface waves.

structed. This in turn may allow conclusions on the surface condition like deposits or disruptions.

In this paper we present analytical solutions for the characteristics of electromagnetic axial surface waves along dielectrically coated conducting cylinders. Dispersion characteristics are derived for TM, TE, and hybrid waves. The effect of losses due to finite conductivity and dielectric losses is particularly considered. Emphasis is placed on gigahertz frequencies and cylinder diameters and layer thicknesses in the centimeter range. As a result we present analytical solutions for dispersion characteristics and attenuation of different wave modes versus frequency and permittivity with a focus on the fundamental $\mathrm{TM}_{00}$ mode which is suitable for wave excitation through a coaxial waveguide structure. Finally a study of the radial power distribution as a function of material parameters is also presented. 
Table 1. Classification of complex waves after Ishimaru (1991).

\begin{tabular}{lllll}
\hline$\beta_{z}$ & $\alpha_{z}$ & $\beta_{x}$ & $\alpha_{x}$ & the term used \\
\hline \multicolumn{3}{l}{ proper waves } \\
\hline+ & 0 & + & 0 & Fast wave (waveguide modes) \\
+ & - & + & + & Backward leaky wave \\
+ & 0 & 0 & + & Trapped surface wave (slow wave) \\
+ & + & - & + & Zenneck wave \\
+ & 0 & - & 0 & Plane-wave incidence \\
\hline improper waves & & \\
\hline+ & - & - & - & \\
+ & 0 & 0 & - & Untrapped surface wave \\
+ & + & + & - & Forward leaky wave \\
\hline
\end{tabular}

\section{Review of complex waves}

As an introduction to the topic of surface waves we give a short review of the properties of complex waves as they were discussed by Ishimaru (1991). We consider a 2-D geometry with infinite extent in cartesian coordinates $x, y$, and $z$. There are two media in the half spaces $x<0$ and $x>0$, respectively. Directions $x$ and $z$ are considered directions of wave propagation. The problem shell not depend on $y$, i.e. $\partial / \partial y=0$ for all quantities. We obtain the following wave equation for a scalar quantity $u(x, z)$ :

$\left(\frac{\partial^{2}}{\partial x^{2}}+\frac{\partial^{2}}{\partial z^{2}}+k^{2}\right) u(x, z)=0$.

It's general solution is given by

$u(x, z)=\mathrm{e}^{-\mathrm{j} k_{x} x-\mathrm{j} k_{z} z}$

where $k_{x}$ and $k_{z}$ are wave numbers and $k_{x, z}=\beta_{x, z}-\mathrm{j} \alpha_{x, z}$. From $k_{x}^{2}+k_{z}^{2}=k^{2}=\omega^{2} \varepsilon \mu-\mathrm{j} \omega \mu \sigma$ and by comparing real parts and imaginary parts we get the relations

$\beta_{x}^{2}-\alpha_{x}^{2}+\beta_{z}^{2}-\alpha_{z}^{2}=\omega^{2} \mu \varepsilon$,

$\beta_{x} \alpha_{x}+\beta_{z} \alpha_{z}=\omega \mu \sigma$.

With $\boldsymbol{\alpha}=\left(\alpha_{x} \alpha_{z}\right)^{\mathrm{T}}$ and $\boldsymbol{\beta}=\left(\beta_{x} \beta_{z}\right)^{\mathrm{T}}$ it follows that in the lossless case $(\sigma=0)$ it is $\boldsymbol{\beta} \cdot \boldsymbol{\alpha}=0$. Several solutions for wave numbers that satisfy the boundary conditions in each point of the interface plane $x=0$ can be found. They may be classified depending on the values of $\alpha_{x, z}$ and $\beta_{x, z}$. An overview is given in Table 1 . Solutions where $\alpha_{x}<0$ will increase exponentially and are therefore unphysical. They are termed improper waves. Even though they are unphysical they yield practically meaningful wave types when the extent of the interface is not infinite but finite.

Let us summarize some of the solutions in Table 1 in more detail starting with the Zenneck wave. It can exist when at least one of the two media is lossy. In this case the dispersion equation may be solved exact and analytically. For a

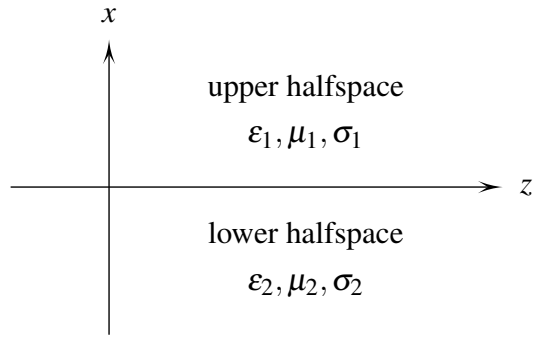

Fig. 2. Zenneck wave guiding geometry.

circular cylindrical interface the solution can only be found numerically. Also for cylinders the solution will depend what is the outer and what is the inner medium. A trivial example is the different nature of a conducting wire and a hollow waveguide. However, even for a plane interface it is found that there exists no solution of TEM type.

Assuming a configuration as shown in Fig. 2 and restricting to TM solutions the following differential equation for $H_{y}$ is found:

$\frac{1}{\sigma+\mathrm{j} \varepsilon \omega} \frac{\partial^{2} H_{y}}{\partial x^{2}}-\left(k_{z}^{2} \frac{1}{\sigma+\mathrm{j} \varepsilon \omega}+\mathrm{j} \mu \omega\right) H_{y}=0$.

It is solved in both half-spaces by the ansatz $H_{y}=C \mathrm{e}^{-\mathrm{j} k_{x} x}$. By further investigating the solution it is readily found that the wave numbers $k_{z}, k_{x, 1}$ and $k_{x, 2}$ correspond to the case of TM incidence at Brewster's angle. In this case Brewster's angle appears to be complex due to the losses in the medium. Hence there is no coincidence between planes of constant amplitude and planes of constant phase planes i.e. the solution represents an inhomogeneous TEM wave.

The trapped surface wave is a solution that is obtained by the assumption of loss-free propagation in $z$ direction. Its phase velocity is given by

$v_{\text {ph }}=\frac{\omega}{\beta_{z}}=\frac{\omega}{k_{z}}=\frac{\omega}{k}$.

Since the phase velocity of a trapped surface wave is smaller than in free space it belongs to the class of slow wave solutions. From

$k_{x}^{2}+\beta_{z}^{2}=k^{2}$

it follows that $k_{x}^{2}=k^{2}-\beta_{z}^{2}<0$. Furthermore, if $k$ and $\beta_{z}$ are real it is necessary that $\operatorname{Re}\left\{k_{x}\right\}=0$ i.e.

$\alpha_{x} \neq 0 \quad \beta_{x}=0$.

An example geometry where trapped surface waves can exist is shown in Fig. 3. For both TE and TM waves the same dispersion relation

$$
\begin{aligned}
& \frac{k_{x, 1}}{\varepsilon_{1}} \tan \left(k_{x, 1} d\right)=\mathrm{j} \frac{k_{x, 0}}{\varepsilon_{0}} \\
& k_{x, 0 / 1}=\sqrt{k_{0,1}^{2}-k_{z}^{2}}
\end{aligned}
$$




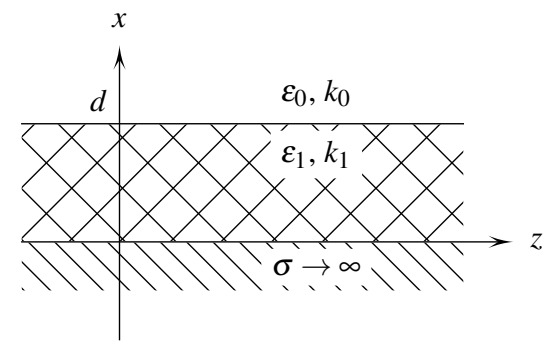

Fig. 3. Slow-wave guiding geometry.

holds. In this case the dispersion relation is transcendental and may be solved e.g. graphically.

\section{Waves along conducting cylinders}

Let us review surface waves in cylindrical structures. To start we discuss conducting cylinders without dielectric coating first. An efficient way to a general solution of the wave equation in cylindrical coordinates is to introduce the Hertz's vector $\boldsymbol{\pi}(\boldsymbol{r}, t)$. It must satisfy the Helmholtz equation

$\Delta \pi+k^{2} \pi=0$.

A practical ansatz in frequency domain is given by

$\boldsymbol{\pi}(\boldsymbol{r}, t)=\boldsymbol{\pi}(\boldsymbol{r}) \mathrm{e}^{\mathrm{j} \omega t}$.

Solutions are obtained by introducing the electric and the magnetic Hertz's vector in the forms

$\boldsymbol{\pi}_{\mathrm{m}}=\pi_{\mathrm{m}}(r, \varphi, z) \boldsymbol{e}_{z} \quad \boldsymbol{\pi}_{\mathrm{e}}=\pi_{\mathrm{e}}(r, \varphi, z) \boldsymbol{e}_{z}$.

It can be shown that the choice of one common direction is sufficient for a complete and general solution. The choice of the direction $\boldsymbol{e}_{z}$ along the cylinder axis is advantageous in this case. By separation of variables, i.e. $\pi_{\mathrm{m}, \mathrm{e}}=R(r) \Phi(\varphi) Z(z)$ solutions of the form

$\pi_{\mathrm{e}, z}=a_{n} \cdot Z_{n}\left(r \sqrt{k^{2}-k_{z}^{2}}\right) \mathrm{e}^{ \pm \mathrm{j} n \varphi} \mathrm{e}^{-\mathrm{j} k_{z} z} \mathrm{e}^{\mathrm{j} \omega t}$ TM waves

$\pi_{\mathrm{m}, z}=b_{n} \cdot Z_{n}\left(r \sqrt{k^{2}-k_{z}^{2}}\right) \mathrm{e}^{ \pm \mathrm{j} n \varphi} \mathrm{e}^{-\mathrm{j} k_{z} z} \mathrm{e}^{\mathrm{j} \omega t}$ TE waves

are found. Here $Z_{n}(x)$ is composed from two particulate solutions of the Bessel differential equation. Considering TM waves along a cylinder with finite conductivity the continuity of fields from (o)utside to (i)nside yields the dispersion equation

$\frac{k_{\mathrm{o}}^{2}}{\mu_{\mathrm{o}} s_{\mathrm{o}}} \frac{\mathrm{H}_{0}^{(1) \prime}\left(s_{\mathrm{o}} r_{0}\right)}{\mathrm{H}_{0}^{(1)}\left(s_{\mathrm{o}} r_{0}\right)}=\frac{k_{\mathrm{i}}^{2}}{\mu_{\mathrm{i}} s_{\mathrm{i}}} \frac{\mathrm{J}_{0}^{\prime}\left(s_{\mathrm{i}} r_{0}\right)}{\mathrm{J}_{0}\left(s_{\mathrm{i}} r_{0}\right)} ; \quad s_{\mathrm{o}, \mathrm{i}}=\sqrt{k_{\mathrm{o}, \mathrm{i}}^{2}-k_{z}^{2}}$.

Since we expect solutions with $k_{z}^{2} \approx k_{\text {out }}^{2}$ we assume that $s_{\mathrm{O}}$ is very small, i.e. $s_{0} \rightarrow 0$. Inside the conducting cylinder the
Table 2. TM dominant wave: phase constant versus conductivity $(r=2.5 \mathrm{~cm}, f=2.41 \mathrm{GHz})$.

\begin{tabular}{ccc}
\hline $\begin{array}{c}\text { Conductivity } \sigma_{\mathrm{i}} \\
\text { (in } \mathrm{m}^{-1} \text { ) }\end{array}$ & $\begin{array}{c}\text { phase constant } \beta_{z} \\
\text { (in } \mathrm{m}^{-1} \text { ) }\end{array}$ & $\begin{array}{c}\text { phase velocity } \\
\text { (in \% of } c_{0} \text { ) }\end{array}$ \\
\hline $10^{1}$ & 51.0368 & 1.0326 \\
$10^{2}$ & 50.6889 & 0.3533 \\
$10^{3}$ & 50.5577 & 0.0946 \\
$10^{4}$ & 50.5227 & 0.0254 \\
$10^{5}$ & 50.5133 & 0.0069 \\
$10^{6}$ & 50.5108 & 0.0019 \\
$10^{7}$ & 50.5101 & 0.0005 \\
\hline
\end{tabular}

wavenumber is predominantly determined by its conductivity $\sigma_{\mathrm{i}}$. Hence, $\operatorname{Re}\left\{s_{\mathrm{i}}\right\}$ will be a large value and $\operatorname{Im}\left\{s_{\mathrm{i}}\right\}$ will be a large and negative value. Therefore the approximations

$\mathrm{J}_{0}^{\prime}(u) / \mathrm{J}_{0}(u)=\mathrm{j} ; \quad \mathrm{H}_{0}(v)=\frac{2 \mathrm{j}}{\pi} \ln \frac{\gamma v}{2 \mathrm{j}} ; \quad \gamma=1.781072 \ldots$.

are justified. They allow for an iterative numerical solution algorithm. An exemplary result for a non-magnetic cylinder in vacuum with radius $r=2.5 \mathrm{~cm}$ at signal frequency $f=$ $2.41 \mathrm{GHz}$ is $\alpha=2.84 \mathrm{~dB} \mathrm{~km}^{-1}$.

In the case of TE waves along a cylinder with finite conductivity one obtains the dispersion relation

$\frac{\mu_{\mathrm{i}}}{s_{\mathrm{i}}} \frac{\mathrm{J}_{0}^{\prime}\left(s_{\mathrm{i}} r_{0}\right)}{\mathrm{J}_{0}\left(s_{\mathrm{i}} r_{0}\right)}=\frac{\mu_{\mathrm{o}}}{s_{\mathrm{o}}} \frac{\mathrm{H}_{0}^{(1) \prime}\left(s_{\mathrm{o}} r_{0}\right)}{\mathrm{H}_{0}^{(1)}\left(s_{\mathrm{o}} r_{0}\right)}$.

In contrast to the TM case the conditions for dominant wave with low attenuation lead to inconsistencies. Hence no TM dominant waves exist. For the properties of possible subsidiary waves with high attenuation it can be said that if $u=r_{0} \sqrt{k_{\mathrm{i}}^{2}-k_{z}^{2}}$ is large then $\operatorname{Im}\{u\}$ must also be large and negative. Then, also $\operatorname{Im}\left\{v=r_{0} \sqrt{k_{\mathrm{o}}^{2}-k_{z}^{2}}\right\}<0$. This indicates exponentially growing fields in radial direction. Therefore it can be concluded that leaky waves exist for sufficiently large values $u$. Tables 2 and 3 show some numerical results for the phase constant of the TM dominant wave for a cylinder with radius $r=2.5 \mathrm{~cm}$ and signal frequency $f=2.41 \mathrm{GHz}$.

Figure 4 shows the numerical result for the attenuation of the TM dominant wave versus frequency for three different radii. The conductivity is $\sigma=10^{7} \mathrm{~S} \mathrm{~m}^{-1}$.

\section{Waves along dielectrically coated cylinders}

Finally, we consider a perfectly conducting circular cylinder coated with a dielectric material. This structure has been studied in the past for larger ratios between wavelength and wire diameter. It is well known as Harms-Goubeau line and it is shown in Fig. 5 together with conical horns as wave 
Table 3. TM dominant wave: phase constant versus radius ( $f=$ $2.41 \mathrm{GHz})$.

\begin{tabular}{ccc}
\hline $\begin{array}{c}\text { Radius } r \\
\text { (in } \mathrm{cm})\end{array}$ & $\begin{array}{c}\text { phase constant } \beta_{z} \\
\text { (in } \mathrm{m}^{-1} \text { ) }\end{array}$ & $\begin{array}{c}\text { phase velocity } \\
\text { (in } \% \text { of } c_{0} \text { ) }\end{array}$ \\
\hline 0.1 & 50.51525 & 0.01066 \\
0.5 & 50.51107 & 0.00240 \\
0.9 & 50.51057 & 0.00140 \\
1.3 & 50.51037 & 0.00100 \\
1.7 & 50.51026 & 0.00078 \\
2.1 & 50.51019 & 0.00064 \\
2.5 & 50.51014 & 0.00055 \\
\hline
\end{tabular}

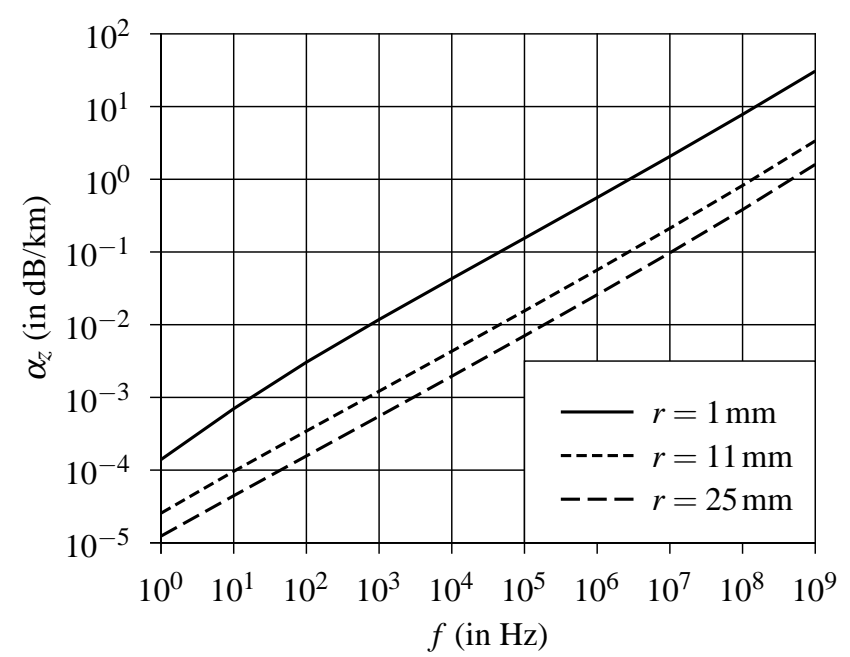

Fig. 4. TM dominant wave: attenuation for $\sigma=10^{7} \mathrm{~S} \mathrm{~m}^{-1}$.

launchers. We can use the same ansatz as in Sect. 3. Again, we skip the lengthy derivation and refer to the derivation of Harms (1907). Because of the more intricate boundary conditions, the eigenvalue equation reads

$\left[\frac{k_{\mathrm{o}}^{2}}{\mu_{\mathrm{o}} s_{\mathrm{o}}} \frac{\mathrm{H}_{n}^{(1) \prime}\left(s_{\mathrm{o}} r_{1}\right)}{\mathrm{H}_{n}^{(1)}\left(s_{\mathrm{o}} r_{1}\right)}\right.$

$\left.-\frac{k_{\mathrm{d}}^{2}}{\mu_{\mathrm{d}} s_{\mathrm{d}}} \frac{\mathrm{J}_{n}^{\prime}\left(s_{\mathrm{d}} r_{1}\right) \mathrm{N}_{n}\left(s_{\mathrm{d}} r_{0}\right)-\mathrm{N}_{n}^{\prime}\left(s_{\mathrm{d}} r_{1}\right) \mathrm{J}_{n}\left(s_{\mathrm{d}} r_{0}\right)}{\left.\mathrm{J}_{\mathrm{d}} r_{1}\right) \mathrm{N}_{n}\left(s_{\mathrm{d}} r_{0}\right)-\mathrm{N}_{n}\left(s_{\mathrm{d}} r_{1}\right) \mathrm{J}_{n}\left(s_{\mathrm{d}} r_{0}\right)}\right]$

$\left[\frac{\mu_{\mathrm{o}}}{s_{\mathrm{o}}} \frac{\mathrm{H}_{n}^{(1) \prime}\left(s_{\mathrm{o}} r_{1}\right)}{\mathrm{H}_{n}^{(1)}\left(s_{\mathrm{o}} r_{1}\right)}\right.$

$\left.-\frac{\mu_{\mathrm{d}}}{s_{\mathrm{d}}} \frac{\mathrm{J}_{n}^{\prime}\left(s_{\mathrm{d}} r_{1}\right) \mathrm{N}_{n}^{\prime}\left(s_{\mathrm{d}} r_{0}\right)-\mathrm{N}_{n}^{\prime}\left(s_{\mathrm{d}} r_{1}\right) \mathrm{J}_{n}^{\prime}\left(s_{\mathrm{d}} r_{0}\right)}{\mathrm{J}_{n}\left(s_{\mathrm{d}} r_{1}\right) \mathrm{N}_{n}^{\prime}\left(s_{\mathrm{d}} r_{0}\right)-\mathrm{N}_{n}\left(s_{\mathrm{d}} r_{1}\right) \mathrm{J}_{n}^{\prime}\left(s_{\mathrm{d}} r_{0}\right)}\right]$

$=\frac{k_{z}^{2} n^{2}}{r_{1}^{2}}\left(\frac{1}{s_{\mathrm{d}}^{2}}-\frac{1}{s_{\mathrm{o}}^{2}}\right)^{2}$.

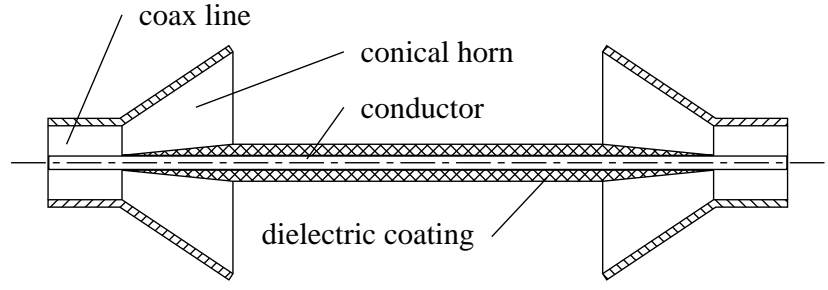

Fig. 5. Structure of a Harms-Goubeau line.

In general, the wave number $k_{z}$ can be complex, which renders it difficult to find a solution. However, if we consider a loss-free dielectric material, then we know that $k_{z}$ is a real number. In addition, we know that the wave number is bounded by $k_{\text {free }}<k_{z}<k_{\mathrm{d}}$. Bessel functions of first and second kind are real for real-valued arguments. The Hankel function of first kind and even orders $n=0,2,4, \ldots$ will give for an argument which has only an imaginary component a number with only an imaginary component. For odd orders $n=1,3,5, \ldots$ the result is purely real. As $s_{\mathrm{o}}$ has only an imaginary component, we know that the contribution of the Hankel functions will be real-valued, like the contribution of the Bessel functions. Hence, to find solutions of the eigenvalue equation, we can just consider the real part of eigenvalue equation.

Furthermore, we see that for $n=0$ the equation decouples into two separate equations, where the first factor of Eq. (18) will result in TM waves and the second factor in TE waves. In general, however, we have hybrid waves. The question is, whether we can find a fundamental wave, i.e. a wave which has no cutoff frequency.

As shown by Fikioris and Roumeliotis (1979), Eq. (18) can be simplified and we obtain formulas for different values of $n$. For $n=0$ or $n=1$, we have $\mathrm{TM}_{0 m}$ waves

$\mathrm{J}_{0}\left(s_{\mathrm{d}} r_{1}\right) \mathrm{N}_{0}\left(s_{\mathrm{d}} r_{0}\right)-\mathrm{N}_{0}\left(s_{\mathrm{d}} r_{1}\right) \mathrm{J}_{0}\left(s_{\mathrm{d}} r_{0}\right)=0$,

$\mathrm{TE}_{0 m}$ waves

$\mathrm{J}_{0}\left(s_{\mathrm{d}} r_{1}\right) \mathrm{N}_{1}\left(s_{\mathrm{d}} r_{0}\right)-\mathrm{N}_{0}\left(s_{\mathrm{d}} r_{1}\right) \mathrm{J}_{1}\left(s_{\mathrm{d}} r_{0}\right)$,

hybrid waves, $\mathrm{HE}_{1 m}$, where $m>1$

$M_{1}=\mathrm{J}_{1}\left(s_{\mathrm{d}} r_{1}\right) \mathrm{N}_{1}\left(s_{\mathrm{d}} r_{0}\right)-\mathrm{N}_{1}\left(s_{\mathrm{d}} r_{1}\right) \mathrm{J}_{1}\left(s_{\mathrm{d}} r_{0}\right)$,

and hybrid waves, $\mathrm{EH}_{1 m}$, where $m \geqslant 1$

$L_{1}=\mathrm{J}_{1}\left(s_{\mathrm{d}} r_{1}\right) \mathrm{N}_{1}^{\prime}\left(s_{\mathrm{d}} r_{0}\right)-\mathrm{N}_{1}\left(s_{\mathrm{d}} r_{1}\right) \mathrm{J}_{1}^{\prime}\left(s_{\mathrm{d}} r_{0}\right)$.

For finding the cutoff frequencies, we have to find the zeros of these equations. Using the formula

$s_{\mathrm{d}}=\omega_{\text {cutoff }} \frac{\sqrt{\varepsilon_{\mathrm{r}, \mathrm{d}} \mu_{\mathrm{r}, \mathrm{d}}-\varepsilon_{\mathrm{r}, \mathrm{o}} \mu_{\mathrm{r}, \mathrm{o}}}}{c}$,

we obtain the cutoff frequencies. The constant $c$ is the speed of light in vacuum. For the TM and TE waves, we can also state a closed form expression. When the frequency is 
Table 4. TM waves: some cutoff frequencies.

\begin{tabular}{|c|c|c|c|c|c|c|c|c|c|}
\hline \multirow[t]{2}{*}{$\varepsilon_{\mathrm{r}, \mathrm{d}}$} & \multicolumn{3}{|c|}{ thickness $d=1 \mathrm{~mm}$} & \multicolumn{3}{|c|}{ thickness $d=5 \mathrm{~mm}$} & \multicolumn{3}{|c|}{ thickness $d=20 \mathrm{~mm}$} \\
\hline & $\begin{array}{c}f_{01} \\
\text { (in } \mathrm{GHz} \text { ) }\end{array}$ & $\begin{array}{c}f_{02} \\
\text { (in } \mathrm{GHz} \text { ) }\end{array}$ & $\begin{array}{c}f_{03} \\
\text { (in } \mathrm{GHz} \text { ) }\end{array}$ & $\begin{array}{c}f_{01} \\
\text { (in } \mathrm{GHz} \text { ) }\end{array}$ & $\begin{array}{c}f_{02} \\
\text { (in } \mathrm{GHz} \text { ) }\end{array}$ & $\begin{array}{c}f_{03} \\
\text { (in } \mathrm{GHz} \text { ) }\end{array}$ & $\begin{array}{c}f_{01} \\
\text { (in } \mathrm{GHz} \text { ) }\end{array}$ & $\begin{array}{c}f_{02} \\
\text { (in } \mathrm{GHz} \text { ) }\end{array}$ & $\begin{array}{c}f_{03} \\
\text { (in } \mathrm{GHz} \text { ) }\end{array}$ \\
\hline 2 & 149.90 & 299.79 & 449.68 & 29.97 & 59.95 & 89.93 & 7.49 & 14.98 & 22.48 \\
\hline 4 & 86.54 & 173.08 & 259.62 & 17.30 & 34.61 & 51.92 & 4.32 & 8.65 & 12.98 \\
\hline 6 & 67.03 & 134.07 & 201.10 & 13.40 & 26.81 & 40.22 & 3.35 & 6.70 & 10.05 \\
\hline 8 & 56.65 & 113.31 & 169.96 & 11.33 & 22.66 & 33.99 & 2.83 & 5.66 & 8.49 \\
\hline
\end{tabular}

Table 5. TE waves: some cutoff frequencies.

\begin{tabular}{|c|c|c|c|c|c|c|c|c|c|}
\hline \multirow[t]{2}{*}{$\varepsilon_{\mathrm{r}, \mathrm{d}}$} & \multicolumn{3}{|c|}{ thickness $d=1 \mathrm{~mm}$} & \multicolumn{3}{|c|}{ thickness $d=5 \mathrm{~mm}$} & \multicolumn{3}{|c|}{ thickness $d=20 \mathrm{~mm}$} \\
\hline & $\begin{array}{c}f_{01} \\
\text { (in } \mathrm{GHz} \text { ) }\end{array}$ & $\begin{array}{c}f_{02} \\
\text { (in } \mathrm{GHz} \text { ) }\end{array}$ & $\begin{array}{c}f_{03} \\
\text { (in } \mathrm{GHz} \text { ) }\end{array}$ & $\begin{array}{c}f_{01} \\
\text { (in } \mathrm{GHz} \text { ) }\end{array}$ & $\begin{array}{c}f_{02} \\
\text { (in } \mathrm{GHz} \text { ) }\end{array}$ & $\begin{array}{c}f_{03} \\
\text { (in } \mathrm{GHz} \text { ) }\end{array}$ & $\begin{array}{c}f_{01} \\
\text { (in } \mathrm{GHz} \text { ) }\end{array}$ & $\begin{array}{c}f_{02} \\
\text { (in } \mathrm{GHz} \text { ) }\end{array}$ & $\begin{array}{c}f_{03} \\
\text { (in } \mathrm{GHz} \text { ) }\end{array}$ \\
\hline 2 & 74.94 & 224.84 & 374.74 & 14.98 & 44.96 & 74.94 & 3.74 & 11.24 & 18.73 \\
\hline 4 & 43.27 & 129.81 & 216.35 & 8.65 & 25.96 & 43.27 & 2.16 & 6.49 & 10.81 \\
\hline 6 & 33.51 & 100.55 & 167.58 & 6.70 & 20.11 & 33.51 & 1.67 & 5.02 & 8.37 \\
\hline 8 & 28.32 & 84.98 & 141.63 & 5.66 & 16.99 & 28.32 & 1.41 & 4.24 & 7.08 \\
\hline
\end{tabular}

large enough, we can use the asymptotic approximation of the Bessel functions. Therefore, we obtain

$\omega_{m, \text { cutoff }}=m \cdot \frac{\pi}{d} \frac{c}{\sqrt{\varepsilon_{\mathrm{r}, \mathrm{d}} \mu_{\mathrm{r}, \mathrm{d}}-\varepsilon_{\mathrm{r}, \mathrm{o}} \mu_{\mathrm{r}, \mathrm{o}}}}$

and

$\omega_{m, \text { cutoff }}=\frac{1}{d}\left(m+\frac{1}{2}\right) \pi \cdot \frac{c}{\sqrt{\varepsilon_{\mathrm{r}, \mathrm{d}} \mu_{\mathrm{r}, \mathrm{d}}-\varepsilon_{\mathrm{r}, \mathrm{o}} \mu_{\mathrm{r}, \mathrm{o}}}}$

for the TM and TE case, respectively, where $d=r_{1}-r_{0}$ is the thickness of the dielectric coating.

Regarding Eq. (19), we find that $s_{\mathrm{d}}=0$ satisfies this equation. Hence, there is a fundamental TM wave, similar to the one we had in the case of the uncoated conducting cylinder. However, by deriving the Eqs. (19) through (22), it appears that for $n=1$ again $s_{\mathrm{d}}=0$ is a valid solution (Fikioris and Roumeliotis, 1979). Therefore, there is also a fundamental hybrid wave $\mathrm{HE}_{11}$ so that on a dielectric coated conducting cylinder two fundamental waves exist. The cutoff frequencies for different configurations are presented in Tables 4, 5 and 6.

Although more waves can exist than just the fundamental $\mathrm{TM}_{00}$ wave, we are interested in its properties as we can easily excite this type of wave with, e.g. a horn structure. In Fig. 6 we can see the dispersion behavior of the fundamental $\mathrm{TM}_{00}$ wave. This wave is propagating on a cylindrical conductor with radius $r=2.5 \mathrm{~cm}$ and which has a dielectric coating with relative permittivity $\varepsilon_{\mathrm{r}}=8$ and thickness $d=5 \mathrm{~mm}$. We can observe that for the lower frequencies the $k_{z} \approx k_{\mathrm{o}}$, while for high frequencies $k_{z} \approx k_{\mathrm{d}}$. This indicates that for high frequencies the wave is guided closer to the conductor, i.e. the wave is contained mostly in the dielectric.

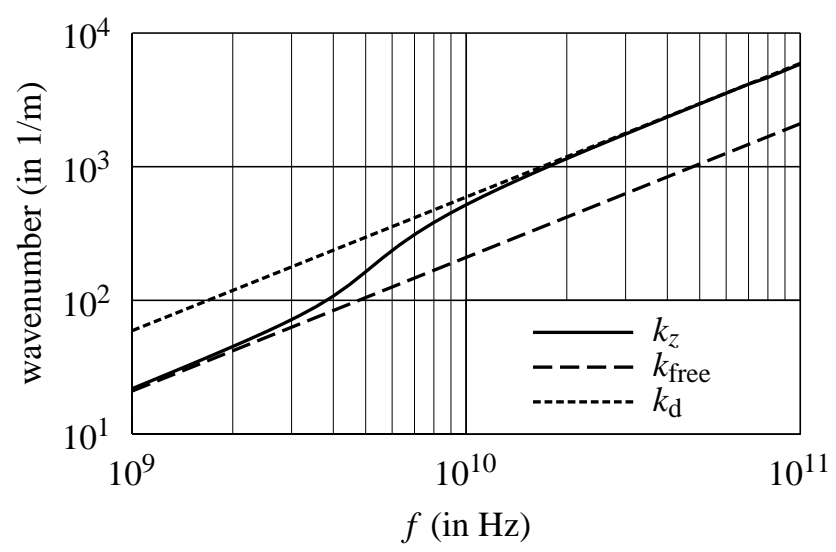

Fig. 6. Dispersion diagram of the $\mathrm{TM}_{00}$ fundamental mode.

The power transported in the dielectric is

$P_{\mathrm{d}}=2 \pi \int_{r_{0}}^{r_{1}} \frac{1}{2} E_{r} H_{\varphi} r \mathrm{~d} r=\frac{k_{z}}{4 \pi \omega \varepsilon_{\mathrm{d}}} \ln \frac{r_{1}}{r_{0}}|I|^{2}$,

while the power transported in the exterior is

$P_{\mathrm{a}}=\pi\left|a_{0}^{\mathrm{a}}\right|^{2} \sqrt{\frac{\varepsilon_{\mathrm{a}}}{\mu_{\mathrm{a}}}}\left|s_{\mathrm{a}}\right|^{2} k_{z} k_{\mathrm{a}} \frac{1}{2 \varsigma_{\mathrm{a}}^{2}} \cdot\left[F\left(\varsigma_{\mathrm{a}} r\right)-F\left(\varsigma_{\mathrm{a}} r_{1}\right)\right]$,

where

$$
\begin{aligned}
F\left(\varsigma_{\mathrm{a}} r\right) & =\left(\varsigma_{\mathrm{a}} r\right)^{2}\left\{\frac{2}{\varsigma_{\mathrm{a}} r} \mathrm{j}_{0}^{(1)}\left(\mathrm{j} \varsigma_{\mathrm{a}} r\right) \mathrm{H}_{1}^{(1)}\left(\mathrm{j} \varsigma_{\mathrm{a}} r\right)\right. \\
& \left.+\left[\mathrm{H}_{0}^{(1)}\left(\mathrm{j} \varsigma_{\mathrm{a}} r\right)\right]^{2}+\left[\mathrm{H}_{1}^{(1)}\left(\mathrm{j} \varsigma_{\mathrm{a}} r\right)\right]^{2}\right\}
\end{aligned}
$$


Table 6. Hybrid waves: some cutoff frequencies.

\begin{tabular}{|c|c|c|c|c|c|c|c|c|c|}
\hline \multirow[t]{2}{*}{$\varepsilon_{\mathrm{r}, \mathrm{d}}$} & \multicolumn{3}{|c|}{ thickness $d=1 \mathrm{~mm}$} & \multicolumn{3}{|c|}{ thickness $d=5 \mathrm{~mm}$} & \multicolumn{3}{|c|}{ thickness $d=20 \mathrm{~mm}$} \\
\hline & $\begin{array}{c}f_{12} \\
\text { (in GHz) }\end{array}$ & $\begin{array}{c}f_{13} \\
\text { (in } \mathrm{GHz} \text { ) }\end{array}$ & $\begin{array}{c}f_{14} \\
\text { (in } \mathrm{GHz} \text { ) }\end{array}$ & $\begin{array}{c}f_{12} \\
\text { (in } \mathrm{GHz} \text { ) }\end{array}$ & $\begin{array}{c}f_{13} \\
\text { (in } \mathrm{GHz} \text { ) }\end{array}$ & $\begin{array}{c}f_{14} \\
\text { (in } \mathrm{GHz} \text { ) }\end{array}$ & $\begin{array}{c}f_{12} \\
\text { (in } \mathrm{GHz} \text { ) }\end{array}$ & $\begin{array}{c}f_{13} \\
\text { (in } \mathrm{GHz} \text { ) }\end{array}$ & $\begin{array}{c}f_{14} \\
\text { (in } \mathrm{GHz} \text { ) }\end{array}$ \\
\hline 2 & 149.90 & 299.79 & 449.69 & 30.01 & 59.97 & 89.95 & 7.59 & 15.03 & 22.51 \\
\hline 4 & 86.54 & 173.08 & 259.62 & 17.33 & 34.62 & 51.93 & 4.38 & 8.68 & 13.00 \\
\hline 6 & 67.03 & 134.07 & 201.10 & 13.42 & 26.82 & 40.22 & 3.39 & 6.72 & 10.07 \\
\hline 8 & 56.65 & 113.31 & 169.96 & 11.34 & 22.66 & 33.99 & 2.86 & 5.68 & 8.51 \\
\hline
\end{tabular}

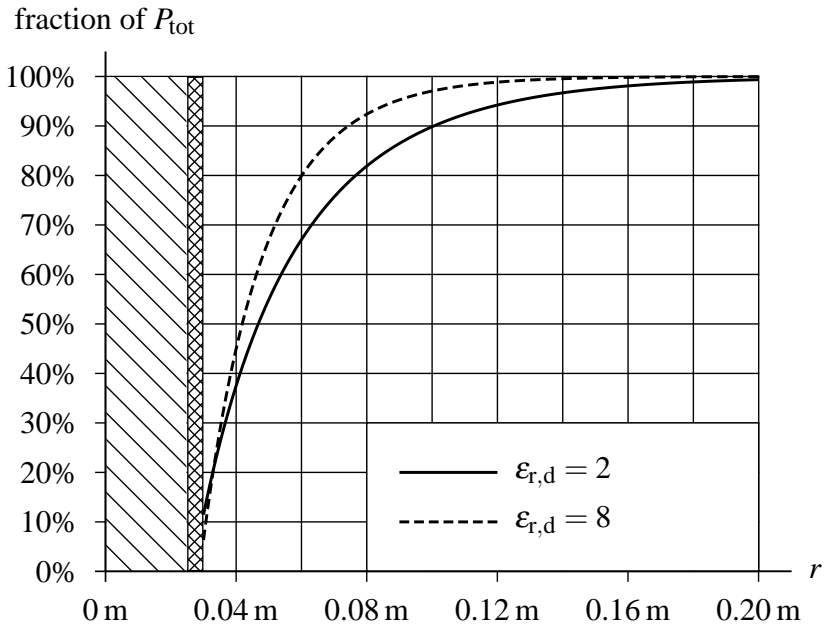

Fig. 7. Spatial power distribution of the $\mathrm{TM}^{00}$ fundamental mode, shown is the fraction of the total power $P_{\text {tot }}$ that is guided inside a cylindrical surface with radius $r$. The thickness of the dielectric layer is $d=5 \mathrm{~mm}$.

and $\varsigma_{\mathrm{a}}=\sqrt{k_{z}^{2}-k_{\mathrm{a}}^{2}}$ as given by Goubau (1950). The spatial power distribution for a conductor with radius $2.5 \mathrm{~cm}$ and dielectric coating with thickness $d=5 \mathrm{~mm}$ and relative permittivities $\varepsilon_{\mathrm{r}}=2$ and $\varepsilon_{\mathrm{r}}=8$, respectively, is shown in Fig. 7 . More than $75 \%$ of the power is contained within a $5 \mathrm{~cm}$ radius when the relative permittivity is $\varepsilon_{\mathrm{r}}=8$.

\section{Conclusions}

In this paper the fundamental properties of surface waves along conducting cylinders have been investigated for cylinder diameters in the centimeter range and frequencies in the gigahertz range and higher. For cylinders without additional dielectric coating the $\mathrm{TM}_{00}$ dominant wave (axial Sommerfeld wave) has been considered. Its dispersion and attenuation characteristics have been derived and the effect of cylinder radius and conductivity on the phase constant has been studied. As an exemplary result on the guiding properties it can be concluded that for a radius $r=2.5 \mathrm{~cm}$, frequency $f=2.41 \mathrm{GHz}$, and conductivity $\sigma=10^{7} \mathrm{~S} \mathrm{~m}^{-1} 75 \%$ of the total power are carried within a radius of $r=1 \mathrm{~m}$.
For the case of conducting cylinders with additional dielectric coating on their surface $\mathrm{TM}_{0 m}, \mathrm{TE}_{0 m}$, and hybrid waves have been studied. We computed cutoff frequencies for various material parameters and derived and approximate formula for the wave attenuation assuming perfect conductor and dielectric losses. Again the effect of cylinder radius and permittivity has been studied. As an exemplary result on the guiding properties with additional dielectric coating it can be said that for a cylinder radius $r=2.5 \mathrm{~cm}$, frequency $f=2.41 \mathrm{GHz}$, and dielectric coating with relative permittivity $\varepsilon_{\mathrm{r}}=8$ and thickness $d=1 \mathrm{~mm} 75 \%$ of the total power are carried within a radius of $r=10 \mathrm{~cm}$.

\section{References}

Fikioris, J. G. and Roumeliotis, J. A.: Cutoff Wavenumbers of Goubau Lines, IEEE T. Microw. Theory, 27, 570-573, 1979.

Goubau, G.: Surface Waves and Their Application to Transmission Lines, J. Appl. Phys., 21, 1119-1128, 1950.

Goubau, G.: Single-Conductor Surface-Wave Transmission Lines, Proceedings of the IRE, 39, 619-625, 1951.

Goubau, G.: Cylindrical Dielectric Waveguide Modes, IRE Transactions on Antennas and Propagation, 7, 140-146, 1959.

Harms, F.: Elektromagnetische Wellen an einem Draht mit isolierender zylindrischer Hülle, Ann. Phys.-Berlin, 328, 44-60, 1907.

Ishimaru, A.: Electromagnetic Wave Propagation, Radiation and Scattering, Prentice Hall, Englewood Cliffs, New Jersey, 10th Edn., 1991.

Roberts Jr., T. E.: Theory of the Single-Wire Transmission Line, J. Appl. Phys., 24, 57-67, 1953.

Schelkunoff, S. A.: Anatomy of Surface waves, IEEE Transactions on Antennas and Propagation, 7, 133-139, 1959.

Snitzer, E.: Cylindrical Dielectric Waveguide Modes, J. Opt. Soc. Am. (1917-1983), 51, 491-498, 1961. 